\title{
Combining fNIRS and EEG to Improve Motor Cortex Activity Classification during an Imagined Movement- Based Task
}

\author{
Darren J. Leamy, Rónán Collins, and Tomas E. Ward \\ Biomedical Engineering Research Group, \\ Department of Electronic Engineering, \\ NUI Maynooth, \\ Ireland. Adelaide \& Meath Hospital, Tallaght, \\ Dublin 24, Ireland \\ dleamy@eeng.nuim.ie, \\ ronan.collins2@amnch.ie, \\ tomas.ward@eeng.nuim.ie
}

\begin{abstract}
This work serves as an initial investigation into improvements to classification accuracy of an imagined movement-based Brain Computer Interface (BCI) by combining the feature spaces of two unique measurement modalities: functional near infrared spectroscopy (fNIRS) and electroencephalography (EEG). Our dual-modality system recorded concurrent and co-locational hemodynamic and electrical responses in the motor cortex during an imagined movement task, participated in by two subjects. Offline analysis and classification of fNIRS and EEG data was performed using leave-one-out cross-validation (LOOCV) and linear discriminant analysis (LDA). Classification of 2dimensional fNIRS and EEG feature spaces was performed separately and then their feature spaces were combined for further classification. Results of our investigation indicate that by combining feature spaces, modest gains in classification accuracy of an imagined movement-based BCI can be achieved by employing a supplemental measurement modality. It is felt that this technique may be particularly useful in the design of BCI devices for the augmentation of rehabilitation therapy.
\end{abstract}

\section{Introduction}

A brain-computer interface (BCI) is a system for generating computer control signals based on changes in monitored brain activity [1], [2]. BCIs have been used for many diverse reasons, such as for allowing tetraplegics to interact with computers [3], amputees to control prosthetic robotic limbs [4] and healthy subjects to control computer interfaces through thought alone [5]. Our research interest however is in the use of a BCI for stroke rehabilitation. We aim to use this system to circumvent the stroke affected area of a patient's brain by encouraging the neuroplastic process.

Neuroplasticity is the process by which the human brain physically alters neuronal connections within itself in order to adapt to sensory input. During the $20^{\text {th }}$ century it was widely believed that physical changes in the adult brain as a response to sensory 
input were impossible. Research articles challenging this consensus appeared during the past decade and began changing this belief about the brain. Researchers thus began exploring the capabilities and possibilities of a brain that can physically adapt to changing sensory inputs. Research into neuroplasticity has found that dyslexia in children can be treated [6], discovered that blind people use the visual cortex to help process other information [7] and that a musician can improve their musical abilities through mental rehearsal [8] - each of these as a result of a physically changing brain.

In cases where a stroke sufferer has lost the use of a limb, the neuroplastic process is capable of reassigning a different area of the brain to take over from the strokedamaged area [9]. In certain stroke cases, it may still be possible to record a patient's attempt to move a stroke-affected limb in the motor cortex. A stroke patient's attempts to move a stroke-affected limb may be similar to a healthy subject imagining limb movement when the the motor cortex is still intact (as can be the case for lacunar strokes). For this reason, this paper investigates imagined movement-related activity in the motor cortex of healthy subjects.

BCIs require a method for monitoring brain activity, from the analysis of which external control signals are generated. Our research is in stroke rehabilitation so our subjects may be weak, have low mobility and may move during measurement. We therefore use two measurement modalities that are portable and relatively inexpensive: functional near infrared spectroscopy (fNIRS) and electroencephalography (EEG). Both of these modalities have unique advantages and they do not interfere with each other. EEG has very high temporal resolution whereas fNIRS is not affected by electromagnetic interference and is not as susceptible to movement artefact as EEG. By using both modalities on the same area of cortex, extra information about the cortical activity can be recorded. As this implements a combined electrical and hemodynamic recording of cortical activity, we are making direct observations of neurovascular coupling. Such information may prove to be vital for our research into stroke rehabilitation.

\section{1 fNIRS}

fNIRS is a measurement modality based on changing concentrations of oxyhaemoglobin $(\mathrm{HbO})$ and deoxy-haemoglobin $(\mathrm{HbR})$ in cortical areas of the brain. Multiple wavelengths of light in the red to near-infrared range of the electromagnetic spectrum $(620 \mathrm{~nm}-1200 \mathrm{~nm})$ are emitted into the scalp of a subject from the surface of the head from an fNIRS "source". Light incident on the head disperses through the biological tissue, a portion of which exits the head again after passing through cortical areas of the brain, where the chromophores $\mathrm{HbO}$ and $\mathrm{HbR}$ are present. For a given entry and exit point on the scalp, the photons are known to have followed a roughly banana-shaped path through the head, known as the "photon path" [10]. The mean depth of the photon path is related to the physical distance between the points of entry and exit on the scalp. The intensity of the wavelengths of light transmitted through the head is measured with a fNIRS "detector", which is then used to infer time-changing concentrations of $\mathrm{HbO}$ and $\mathrm{HbR}$ along the photon path. This is done using the modified Beer-Lambert law, which describes optical attenuation in a highly-scattering medium [11]: 


$$
\text { Attenuation }(O D)=\log _{10} \frac{l_{0}}{1}=\alpha C B L+G
$$

where OD is the optical densities, $\mathrm{I}_{0}$ is the incident light intensity, $\mathrm{I}$ is the transmitted intensity, $\alpha$ is the absorption coefficient of the chromophore, $\mathrm{c}$ is the concentration of the chromophore, $\mathrm{B}$ is the differential pathlength factor, $\mathrm{L}$ is the distance between the source and detector and $\mathrm{G}$ is a term to account for scattering loss. If measurements are only made of the changes in light attenuation then $\mathrm{B}, \mathrm{L}$ and $\mathrm{G}$ remain constant and changes in $\mathrm{HbO}$ and $\mathrm{HbR}$ concentration can be derived from the expression:

$$
\delta c=\frac{\partial O D}{\alpha B L}
$$

A typical hemodynamic activation response is an initial decrease in $\mathrm{HbO}$ and increase in $\mathrm{HbR}$ followed by a large increase in $\mathrm{HbO}$ and a decrease in $\mathrm{HbR}$ while the cortex is active. When the cortex is at rest, $\mathrm{HbO}$ and $\mathrm{HbR}$ concentrations return to baseline levels. These changes in $\mathrm{HbO}$ and $\mathrm{HbR}$ concentration are used to determine hemodynamically whether an area of cortex is active or not.

\subsection{EEG}

Non-invasive EEG is the measurement of the spatially integrated dendritic activity of similarly oriented neurons near the surface of the brain. EEG features a spectral structure which changes locally in response to neuronal activity. Spectral power changes in the EEG which occur in temporal relation to subject engagement with a task are known as Event Related Synchronisation (ERS) and Event Related Desynchronisation (ERD). The particular ERS/ERD responses in the motor cortex to motor tasks have been detailed elsewhere [12]. Immediately before a subject engages with a motor task, the motor cortex EEG exhibits a suppression of power in the $\mu$ frequency range (8-12 Hz), known as Pre Movement Mu Desynchronisation (PMMD) [13]. Similarly, when a subject rests from motor activity, an increase of power in the $\beta$ frequency range $(12-30 \mathrm{~Hz})$ is observed shortly after, known as Post Movement Beta Synchronisation (PMBS) [14]. These changes in spectral power are used to determine electrical changes in motor cortex activity associated with movement and imagined movement.

\section{Methodology}

\subsection{Subjects}

Two healthy individuals participated in the study. Subject A was 38 years old and left-handed. Subject B was 26 years old and right-handed. Both participants had normal or corrected vision. Neither participant had consumed any stimulant prior to the experiment. Each participant gave informed oral consent. The experiment was approved by the ethics board of the National University of Ireland Maynooth.

\subsection{Experimental Procedure}

The subjects were seated in a comfortable chair facing a computer monitor with their feet flat on the floor and their arms resting on armrests. Instructions were delivered 
visually via a computer monitor diagonally measuring $43 \mathrm{~cm}$ and positioned $80 \mathrm{~cm}$ from the subject's eyes, centred at eye-level. Instruction presentation and trigger signal generation were carried out with custom software written using $\mathrm{C \#}$ and the .NET framework. Trigger signals were recorded simultaneously by both the fNIRS and EEG systems.

Each subject completed 40 experimental trials, during which instructions were presented to the subject. Before instruction periods began there was a 10 second wait during which the computer screen was blank. Two types of instruction period were used - an activity period during which the screen displayed the message "Imagine Movement" and a rest period during which the screen displayed the message "Relax". Instruction periods lasted 10 seconds, facilitating a total experimental time of 410 seconds.

Prior to the commencement of the experiment, the subject was handed a fist-sized soft ball and asked to practice squeezing the ball with their dominant hand for one minute. The subject was instructed to imagine squeezing the ball during an activity instruction period. Subjects were told to not make any actual movements. Subjects were instructed to stop imagining the movement during a rest instruction period.

\subsection{Signal Acquisition}

Multichannel fNIRS and EEG systems were implemented concurrently to record both hemodynamic and electrical responses in the motor cortex of the subjects during experiments. Three fNIRS sources, three fNIRS detectors and seven EEG electrodes were arranged in a unique montage on a custom-made head mount. The head mount was made of low-density polythene backed by polyurethane foam. fNIRS sources and detectors were positioned with $3 \mathrm{~cm}$ spacing, with the EEG electrodes positioned at the mid-way point of each pair of adjacent fNIRS sources and detectors. fNIRS acquisition was performed with a TechEn CW6 system (TechEn Inc., MA, USA) using wavelengths of $690 \mathrm{~nm}$ and $830 \mathrm{~nm}$. fNIRS data was digitally sampled at 25 Hz. EEG acquisition was performed with a BioSemi Active Two system (BioSemi Inc., The Netherlands). DC-coupled EEG data was digitally sampled at $2048 \mathrm{~Hz}$.

Figure 1 shows the layout of the fNIRS-EEG montage. fNIRS source positions are labelled S1-S3, fNIRS detector positions are labelled D1-D3 and EEG electrode positions are labelled E1-E7. Seven channels of fNIRS-EEG were recorded. The fNIRS sources and detectors and EEG electrodes used for each channel are displayed in Table 1. During experiments, the fNIRS-EEG montage was centred over the subject's dominant-side motor cortex (C4 of the 20/20 system for left-handed Subject A and C3 for right-handed Subject B). Figure 2 shows the orientation of the montage in place on Subject A's head.

\subsection{Signal Processing and Feature Extraction}

fNIRS. The fNIRS system records raw light intensity signals. These are first converted to changes in optical density $(\Delta \mathrm{OD})$ and then, using the modified BeerLambert law, to changes in concentration $(\Delta \mathrm{HbO}$ and $\Delta \mathrm{HbR})$. 
Table 1. Channel fNIRS and EEG designations

\begin{tabular}{|c|c|c|c|}
\hline \multirow{2}{*}{$\begin{array}{c}\text { Channel } \\
\text { Num }\end{array}$} & \multicolumn{2}{|c|}{ fNIRS } & \multirow{2}{*}{ EEG } \\
\cline { 2 - 3 } & Src & Det & \\
\hline 1 & S1 & D1 & E1 \\
\hline 2 & S1 & D2 & E2 \\
\hline 3 & S2 & D1 & E3 \\
\hline 4 & S2 & D2 & E4 \\
\hline 5 & S3 & D2 & E5 \\
\hline 6 & S2 & D3 & E6 \\
\hline 7 & S3 & D3 & E7 \\
\hline
\end{tabular}

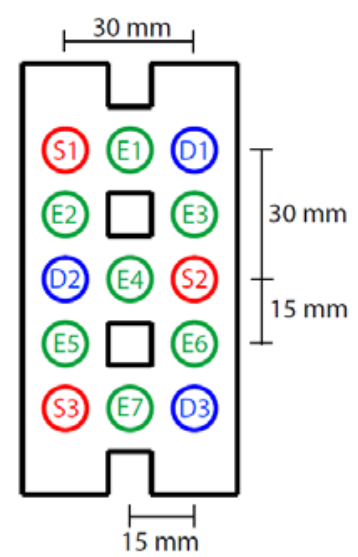

Fig. 1. fNIRS-EEG montage

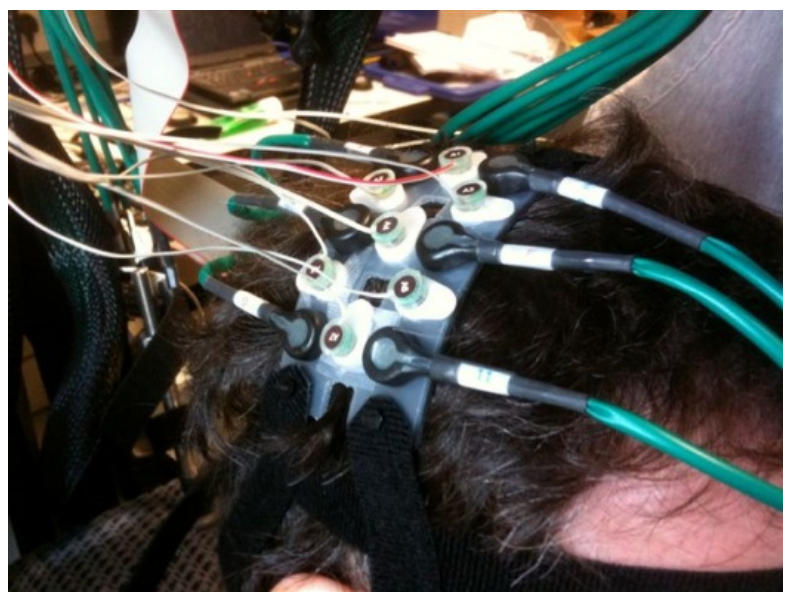

Fig. 2. Dual fNIRS-EEG module over subject's motor cortex

The raw intensity signals were initially low-pass filtered with a cut-off frequency of $0.5 \mathrm{~Hz}$ to remove high-frequency components, such as those associated with the cardiac cycle. Next, the intensity signals are normalised using their mean amplitude over the entire recording. The normalised intensity signals are then high-pass filtered with a cut-off frequency of $0.01 \mathrm{~Hz}$ to remove baseline drift and Mayer wave interference. $\triangle \mathrm{OD}$ signals are obtained by taking the negative logarithm of this filtered signal. 4th order Butterworth filters were used for all filtering steps.

The modified Beer-Lambert law is then used to find the $\Delta \mathrm{HbO}$ and $\Delta \mathrm{HbR}$ signals from the $\triangle \mathrm{OD}$ signals for each channel. Once the source-detector separation for the channel, the extinction coefficients for both the $690 \mathrm{~nm}$ and $830 \mathrm{~nm}$ light in $\mathrm{HbO}$ and $\mathrm{HbR}$ and the differential pathlength factor are known, the operation involves a simple matrix inversion and multiplication to obtain the $\triangle \mathrm{HbO}$ and $\Delta \mathrm{HbR}$ signals. $\mathrm{A}$ differential pathlength factor of 5.93 was used (in accordance with the literature [15]). 
The $\Delta \mathrm{HbO}$ and $\triangle \mathrm{HbR}$ data was then separated into individual 10 second trials. During each trial, the change in average amplitude of the $\Delta \mathrm{HbO}$ and $\Delta \mathrm{HbR}$ signals from the initial 5 seconds to the subsequent 5 seconds was used as the feature to train and test the classifier.

EEG. The EEG data was first high-pass filtered with a cut-off frequency of $1 \mathrm{~Hz}$ to remove DC and near-DC components. The EEG data was then analysed to identify the frequencies at which PMMD and PMBS occurred for the different events. The events were "imagined movement onset" and "imagined movement offset", which coincide with a change in on-screen instruction to the subject. The frequencies at which ERD and ERS occurred were identified by visual inspection of the average FFT plots for the reference and activity periods for both events.

The reference period was chosen to be between 4.5 and 3.5 seconds before both types of event. For an imagined movement onset event, the activity window was chosen to be from between 0 and 1 second after the event. For an imagined movement offset event, the activity window was chosen to be from between 0.5 and 1.5 seconds after the event. The activity windows were chosen because of the expected timings of PMMD and PMBS. The raw EEG is filtered for the identified ranges of PMMD and PMBS. These $\mu$-range and $\beta$-range signals are then squared to get power signals.

The change in $\mu$-range power and $\beta$-range power from reference window to activity window were used as features of EEG activity for classification.

\subsection{Classification}

The goal of classification is to decode the subject's current state based on the features extracted from the fNIRS and EEG responses. The classifier attempted to classify features into one of two classes: 'active' and 'rest'. We employed the Linear Discriminant Analysis (LDA) classifier and calculated classification accuracy via Leave-One-Out Cross Validation (LOOCV), as we had employed previously [16]. In particular, for a total of $N$ trials of data, $N-1$ trials were used for training the classifier and the remaining trial was used for testing. This was repeated $N$ times with each trial used for testing once. Accuracy was measured as the amount of correct classifications over $N$ trials. Following classification of the fNIRS and EEG 2-dimensional feature spaces, the individual feature spaces were combined into an all-encompassing 4dimensional feature space. Every trial of data thus had 4 features available for classification - change in $\mathrm{HbO}$ over the trial, change in $\mathrm{HbR}$ over the trial, change in $\mu$-range power at the start of the trial and change in $\beta$-range power at the start of the trial. This combined 4-dimensional feature space was also classified using LDA and LOOCV.

\subsection{Results}

The classification results are presented in Table 2. Shown are the classification accuracies of the classifier when operating on fNIRS features alone, EEG features alone and combined fNIRS/EEG features. 
Table 2. LDA classification results for fNIRS, EEG and combined features

\begin{tabular}{|c|ccc|ccc|}
\hline & \multicolumn{3}{|c|}{ Subject A } & \multicolumn{3}{c|}{ Subject B } \\
Channel & fNIRS & EEG & Dual & fNIRS & EEG & Dual \\
\hline 1 & $59 \%$ & $51 \%$ & $64 \%$ & $64 \%$ & $46 \%$ & $62 \%$ \\
2 & $56 \%$ & $59 \%$ & $67 \%$ & $51 \%$ & $54 \%$ & $59 \%$ \\
3 & $56 \%$ & $54 \%$ & $64 \%$ & $61 \%$ & $41 \%$ & $56 \%$ \\
4 & $69 \%$ & $67 \%$ & $72 \%$ & $64 \%$ & $59 \%$ & $67 \%$ \\
5 & $61 \%$ & $51 \%$ & $72 \%$ & $41 \%$ & $36 \%$ & $46 \%$ \\
6 & $56 \%$ & $77 \%$ & $64 \%$ & $74 \%$ & $59 \%$ & $69 \%$ \\
7 & $56 \%$ & $59 \%$ & $62 \%$ & $15 \%$ & $43 \%$ & $49 \%$ \\
\hline Average & $59 \%$ & $60 \%$ & $66 \%$ & $53 \%$ & $48 \%$ & $58 \%$ \\
\hline
\end{tabular}

\section{Discussion}

Our results demonstrate that through combining fNIRS and EEG features into a single fNIRS-EEG feature space, an increase in classification accuracy of imagined movement can be obtained. The two experimental subjects had very different classification accuracies of the cortical activity. For Subject A, all but one channel made gains in classification accuracy by combining feature spaces. For Subject B, however, combining feature spaces often results in a classification accuracy result intermediate to those of fNIRS and EEG alone.

From these results, it appears that when fNIRS and EEG classification is reasonably accurate, the combined classification result tends to be higher than both. When either fNIRS or EEG classification accuracy is good and the other is not, combining feature spaces seems to result in intermediate classification accuracy. It is also worth pointing out that when both fNIRS and EEG classification was poor, combining feature spaces resulted in better performance.

These results show that combining fNIRS and EEG feature spaces can results in higher classification accuracy. This is of more importance to improving an fNIRS system than an EEG system. Improving EEG classification accuracy can be accomplished by increasing the density of electrodes over a cortical area. However, fNIRS has a limit to the proximity of source-detector pairs. An fNIRS detector can suffer from "source-blinding" if sources are placed too close, even if that detector is not intentioned to record light from that source. Therefore, for the sole purposes of improving classification accuracy, supplementing an fNIRS system with EEG data is more useful than adding fNIRS to an EEG system.

An advantage of a dual-modality system such as this one is that for the same measurement space on the head, more information about the underlying neurovascular relationship is being recorded. An EEG of fNIRS alone system can only record the electrical or hemodynamic response in an area of cortex. Our system records fNIRS and EEG but also records information about the relationship between them, even if we do not yet fully understand that relationship.

We expect a similar improvement in classification accuracy when using dry EEG electrodes instead of the standard wet electrodes used here. Dry electrodes have much 
lower signal-to-noise ratio (SNR), so combining a dry EEG set-up with fNIRS could help offset the poor SNR. We expect a completely dry fNIRS-EEG system would significantly reduce set-up time, reduce subject discomfort and have similar classification performance to a wet electrode EEG system.

\section{Conclusion}

Investigation into dual-modality measurement is of importance to BCI researchers due to the potential gains in classification accuracy while utilising the same area of cortex. This work has demonstrated that improvements to imagined-movement based BCIs are possible by implementing multi-modal measurements. We believe this research will lead to more accurate BCIs and smaller measurement devices.

Acknowledgements. The authors gratefully acknowledge the contribution of Science Foundation Ireland: Research Frontiers Program 2009, Grant No. 09/RFP/ECE2376.

\section{References}

1. Angelakis, E., et al.: Brain-computer interface: a reciprocal self-regulated neuromodulation. Acta Neurochir. Suppl. 97, 555-559 (2007)

2. Wolpaw, J.R., et al.: Brain-computer interfaces for communication and control. Clinical Neurophysiology 113(6), 767-791 (2002)

3. Conradi, J., et al.: Brain-Computer Interfacing in Tetraplegic Patients with High Spinal Cord Injury. International Journal of Bioelectromagnetism 11(2), 65-68 (2009)

4. Hochberg, L.R., et al.: Neuronal ensemble control of prosthetic devices by a human with tetraplegia. Nature 442, 164-171 (2006)

5. Scherer, R., et al.: The Self-Paced Graz Brain-Computer Interface: Methods and Applications. Comput. Intell. Neurosci., 79826 (2007)

6. Meyler, A., et al.: Modifying the brain activation of poor readers during sentence comprehension with extended remedial instruction: A longitudinal study of neuroplasticity. Neuropsychologia 46(10), 2580-2592 (2008)

7. Burton, H.: Visual Cortex Activity in Early and Late Blind People. The Journal of Neuroscience 23(10), 4005-4011 (2003)

8. Pascual-Leone, A., et al.: Modulation of muscle responses evoked by transcranial magnetic stimulation during the acquisition of new fine motor skills. J. Neurophysiol. 74(3), 10371045 (2005)

9. Johansson, B.B.: Brain Plasticity and Stroke Rehabilitation: The Willis Lecture. Stroke 31, 223-230 (2000)

10. Okada, E., et al.: Theoretical and experimental investigation of near-infrared light propagation in a model of the adult head. Appl. Opt. 36(1), 21-31 (1997)

11. Sassaroli, A., Fantini, S.: Comment on the modified Beer-Lambert law for scattering media. Phys. Med. Biol. 49(14), 255 (2004)

12. Pfurtscheller, G., Lopes da Silva, F.H.: Event-related EEG/MEG synchronization and desynchronization: basic principles. Clinical Neurophysiology 110, 1842-1857 (1999) 
13. Pfurtscheller, G., Aranibar, A.: Evaluation of event-related desynchronization (ERD) preceding and following voluntary self-paced movements. Electroenceph. Clin. Neurophysiol. 46, 138-146 (1979)

14. Pfurtscheller, G., Stancák Jr., A., Neuper, C.: Post-movement beta synchronization. A correlate of an idling motor area? Electroenceph. Clin. Neurophysiol. 98, 281-293 (1996)

15. van der Zee, P., et al.: Experimentally measured optical pathlengths for the adult head, calf and forearm and the head of the newborn infant as a function of inter optode spacing. Adv. Exp. Med. Biol. 316, 143-153 (1992)

16. Leamy, D.J., Ward, T.E.: A novel and concurrent fNIRS/EEG measurement system: design and initial results. In: 2010 Annual International Conference of the IEEE on Engineering in Medicine and Biology Society (EMBC), pp. 4230-4233 (2010) 